\title{
Full counting statistics of noncommuting variables: The case of spin counts
}

\author{
Antonio Di Lorenzo,* Gabriele Campagnano, and Yuli V. Nazarov \\ Kavli Institute of Nanoscience Delft, Faculty of Applied Sciences, Delft University of Technology, Lorentzweg 1, \\ 2628 CJ Delft, The Netherlands
}

(Received 20 October 2005; revised manuscript received 18 January 2006; published 13 March 2006)

\begin{abstract}
We discuss the full counting statistics (FCS) of noncommuting variables with the measurement of successive spin counts in noncollinear directions taken as an example. We show that owing to an irreducible detector back action, the FCS in this case may be sensitive to the dynamics of the detectors, and may differ from the predictions obtained with using a naive version of the projection postulate. We present here a general model of detector dynamics and path-integral approach to the evaluation of FCS. We concentrate further on a simple "diffusive" model of the detector dynamics where the FCS can be evaluated with transfer-matrix method. The resulting probability distribution of spin counts is characterized by anomalously large higher cumulants and substantially deviates from Gaussian statistics.
\end{abstract}

DOI: 10.1103/PhysRevB.73.125311

PACS number(s): $05.60 . \mathrm{Gg}, 72.25 . \mathrm{Ba}$

\section{INTRODUCTION}

There has been a growing interest recently in noise in mesoscopic systems. ${ }^{1}$ Normally, noise is an unwanted feature and, according to classical physics, in principle can be made arbitrarily small by lowering the temperature; according to quantum physics, however, noise is unable to be eliminated due to the intrinsic randomness of elementary processes. Furthermore, noise, rather than being a hindrance, contains valuable information which adds to the one carried by the mean value of the quantity observed. Simple probability distributions, such as, e.g., the Gaussian ones, are determined by the mean values and noise. Even though Gaussian distributions are ubiquitous, there are interesting physical processes which are described by non-Gaussian distributions. Noise alone is not sufficient for the determination of such distributions. One needs to know all the momenta, or equivalently their generating function. Full counting statistics ${ }^{2,3}$ consists in determining the latter.

The FCS approach has been receiving increasing attention from the physics community. Its connection with the formalism of nonequilibrium Green functions ${ }^{4}$ and circuit theory ${ }^{5}$ was established. ${ }^{6}$ It has been used to characterize transport in heterostructures, ${ }^{7}$ shuttling mechanism, ${ }^{8,9}$ charge pumping, ${ }^{10}$ and multiple Andreev reflections. ${ }^{11,12}$ The technique was extended to charge counts in multiterminal structures, ${ }^{13}$ and to spin counts. ${ }^{14}$ The FCS of a general quantum variable was studied, and the necessity of including the dynamics of detectors stressed. ${ }^{15}$

There are some open issues in FCS. The main one concerns whether it is always possible to find a generating function which allows an interpretation in terms of probabilities. Indeed, in Ref. 6 it was found that such an interpretation is not straightforward. This problem was shown to amount to the long-standing question of the nonpositivity of the Wigner distribution. ${ }^{15}$ The lack of a classical interpretation was attributed to the breaking of gauge invariance for the charge degrees of freedom, due to the presence of superconducting terminals. ${ }^{6}$ It is interesting to consider a more general mechanism of gauge invariance breaking which involves spin degrees of freedom. It may be caused either by the presence of ferromagnetic terminals or by subsequent detectors measuring different components of the spin. We shall consider the latter case.

Another issue we want to address is the range of applicability of the projection postulate. Since von Neumann's classic work, ${ }^{16}$ it has been known that Schrödinger's evolution cannot account for the fact that the result of an individual measurement has a unique value, and cannot be described by a superposition. It is necessary to supplement Schrödinger's evolution with an additional evolution (type I in the terminology of von Neumann), projecting the state of the observed system into the eigenstate of the measured observable corresponding to the actual outcome. This can be done at several stages: one could dispense with the description of the measurement, and project the wave function of the system. Alternatively, one may continue the chain by describing the interaction of system and detector, trace out the system's degrees of freedom, and then project the state of the detector. This chain can be continued indefinitely, by skipping the projection of the detector's state, and considering the coupling of the detector with the visible radiation, of the latter with the eye of the observer, etc. So far, it has been implicitly assumed that the predictions of quantum mechanics do not depend on the stage at which one chooses to stop the chain and project. In this work, we shall demonstrate that different statistics are predicted when one projects at the level of the system and at the level of the detector. The reason for this is that, in the example we shall discuss, the quantum dynamics of the detectors cannot be neglected, even after accounting for decoherence.

In addition to being the simplest illustration of noncommuting variables, detection of spin components is a worthy subject in its own right. Spintronics, i.e., the study of how producing, detecting, and manipulating spins, is a rapidly growing field, ${ }^{17}$ which has already found important technological applications. ${ }^{18}$

In this paper, the subsequent detection of noncommuting variables is discussed. The full counting statistics approach allows one to obtain the joint probability distribution for the counts. The noncommutativity of the observed variables manifests itself in the fact that the back action of detectors, 
and their quantum dynamics, must be taken into account. ${ }^{19}$ This remains true when an environment-induced dissipative dynamics for detectors is also included. The reason is that one does not observe one particle at a time, but a flux of particles traversing the detectors at a rate which can be larger than the decoherence rate of the detectors themselves.

The present paper is laid out as follows. In Sec. II the connection of FCS with the density matrix of the detectors is derived, and a general theory of detection of noncommuting variables is presented; a model for the measurement is introduced. In Sec. III, we discuss the case of ideal quantum detectors, having no internal dynamics. We argue that they do not provide a realistic model of detectors because of their long memory. In Sec. IV, we discuss the internal dynamics of the detectors. The fact that detectors are "classical" objects is accounted for by introducing a dissipative dynamics due to their interaction with an environment. Then, since we intend to concentrate on spin counts, in Sec. V we present a model for a spin detector in solid state, relying on spin-orbit interaction. We proceed to Sec. VI by introducing the particular system that we study, namely, two normal reservoirs connected by a coherent conductor. In Sec. VII we give details about the derivation of the FCS for this system, relying on the full quantum-mechanical description of detection process, and we present the results. In Sec. VIII, we discuss the FCS that would be obtained by a naive application of the projection postulate, i.e., by neglecting the quantum dynamics of the detectors. In Sec. IX, we compare the results of the two approaches for the case of one and two detectors in series, and we find that they coincide. In Sec. X, we find a discrepancy between the two approaches when three detectors in series are considered. In particular, we show that both approaches predict the same second-order cross correlators, and that they differ in the prediction of fourth order cumulants $\left\langle\left\langle\sigma_{1}^{2} \sigma_{3}^{2}\right\rangle\right\rangle$. Finally, in Sec. XI, the case of three spin detectors, monitoring the $X, Y$, and $Z$ components of spin current, is presented. The probability distribution for the counts reveals large deviations from the Gaussian distribution.

\section{GENERAL CONSIDERATIONS ABOUT MEASUREMENT}

All the information that we can gain about a system is stored in the density matrix of one or more detectors (denoted by index $a$ ) which have interacted with the system during a time $\tau$. The reduced density matrix is

$$
\hat{\rho}_{\text {det }}(\tau)=\operatorname{Tr}_{\text {sys }}\left\{\mathcal{U}_{\tau, 0} \hat{\rho}(0) \mathcal{U}_{\tau, 0}^{\dagger}\right\}
$$

where $\operatorname{Tr}_{\text {sys }}$ stands for the trace over the degrees of freedom of the measured system, $\hat{\rho}(0)$ is the initial density matrix of system and detectors, and $\mathcal{U}_{\tau, 0}$ is the time evolution operator. We focus on the representation of $\hat{\rho}_{\text {det }}$ in a basis $|\phi\rangle$, $\rho_{\text {det }}^{\phi, \phi^{\prime}}(\tau) \equiv\left\langle\phi\left|\hat{\rho}_{\text {det }}(\tau)\right| \phi^{\prime}\right\rangle$. Here, $|\phi\rangle=\otimes_{a}\left|\phi_{a}\right\rangle$ is a vector in the Hilbert space of the detectors. Since the time evolution is linear, a matrix $\mathcal{Z}_{\mu, \mu^{\prime}}^{\phi, \phi^{\prime}}$ exists such that

$$
\rho_{\mathrm{det}}^{\phi, \phi^{\prime}}(\tau)=\int d \mu d \mu^{\prime} \mathcal{Z}_{\mu, \mu^{\prime}}^{\phi, \phi^{\prime}} \rho_{\mathrm{det}}^{\mu, \mu^{\prime}}(0) .
$$

Thus, given that one knows the initial density matrix of the detectors, $\mathcal{Z}$ contains all the information one can extract from the measurement. However, part of this information gets lost: we can only know the diagonal elements of the density matrix in a particular basis, identified by the pointer states of the detectors. These states, which will be denoted by $|N\rangle$, correspond to the detectors indicating the values $\left\{N_{a}\right\}$, and are individuated by the property that, if one prepares the detector in a generic state identified by a density matrix $\rho_{\text {det }}^{N, N^{\prime}}$, and then lets the environment act on it, the offdiagonal elements of the density matrix in the basis $|N\rangle$ will go to zero with an exponential decay. We point out that this does not dispense us from invoking a projection at some point. The presence of the environment explains how the ensemble averaged density matrix reduces to diagonal form in the basis of pointer states, but it does not explain how the density matrix of the subensemble corresponding to an outcome $N_{a}$ purifies to the state $\left|N_{a}\right\rangle$. This requires invoking the projection postulate for the detector or, equivalently, an evolution dictated by the rules of the Bayesian approach ${ }^{20}$ or of the quantum trajectory ${ }^{21}$ one.

The quantity accessible to observation is the probability to find the detectors in states $\left|N_{a}\right\rangle$, after a time $\tau$. It is given by

$$
P_{\tau}(N)=\left\langle N\left|\hat{\rho}_{\text {det }}(\tau)\right| N\right\rangle .
$$

If off-diagonal elements of the detector's density matrix decay instantaneously, $P_{\tau}(N)$ depends only on the probabilities at a time immediately preceding $\tau, P_{\tau-d t}(N)$, and the process is Markovian.

Now, let us consider the operators $\hat{K}_{a}$ corresponding to the read-out variables of the detectors. Their eigenstates are $\left|N_{a}\right\rangle$, where $N_{a}$ indicates an integer which is proportional to $K_{a}$. The proportionality constant is provided below. Let us also introduce the conjugated operators $\hat{V}_{a},\left[\hat{K}_{a}, \hat{V}_{b}\right]=i \delta_{a b} \hbar$, and their eigenstates $\left|\phi_{a}\right\rangle$, with $\phi_{a}$ dimensionless quantitities proportional to $V_{a}$. If we insert to the left and to the right of $\hat{\rho}_{\text {det }}$ in the right-hand side (RHS) of Eq. (1) the identity (in the detectors' Hilbert space) in the form $\mathcal{I} \propto \int(d \phi / 2 \pi)|\phi\rangle\langle\phi|$, we obtain

$$
P_{\tau}(N)=\int \frac{d \phi^{+}}{2 \pi} \frac{d \phi^{-}}{2 \pi} \exp \left[-\frac{i}{\hbar}\left(\phi^{+}-\phi^{-}\right) \cdot N\right] \rho_{\mathrm{det}}^{\phi^{-}, \phi^{+}}(\tau) .
$$

We used the shorthand $\phi N \equiv \Sigma_{a} \phi_{a} N_{a}$. We change variables according to $\phi^{ \pm}=(\Phi \pm \phi) / 2$. Here, $\Phi$ and $\phi$ are the classical and quantum part of the field, respectively. This terminology reflects the fact that fluctuations of $\Phi$ are set by the temperature, while fluctuations of $\phi$ depend on $\hbar$, as we shall prove in Sec. IV. The time evolution depends on the Hamiltonians of the system and the detectors, and on their interaction. We focus on the detection of internal degrees of freedom of a system whose center of mass coordinate $\mathbf{x}$ is not affected by the presence of the detectors. We consider several detectors 
in series along the path $\mathbf{x}(t)$. We take the interaction to be of the form $H_{\mathrm{int}}=\Sigma_{a} H_{\mathrm{int}}^{a}$ with

$$
H_{\mathrm{int}}^{a}=-\alpha_{a}(\mathbf{x}) \lambda_{a} \hat{V}_{a} \hat{J}_{a}
$$

where $\mathbf{x}$ is the coordinate of the wave packet, $\lambda_{a}$ coupling constants depending on the actual detection setup, $\hat{J}_{a}$ is an operator on the Hilbert space of the system's degrees of freedom, and $\alpha_{a}(\mathbf{x})$ is a function which is unity inside the sensible area of the $a$ th detector and zero outside. For a onedimensional motion, e.g., we would have $\alpha(x)=\theta(x$ $\left.-X^{(\mathrm{in})}\right) \theta\left(X^{(\mathrm{fin})}-x\right)$, where $X^{(\mathrm{in})}$ and $X^{(\mathrm{fin})}$ are the coordinates delimiting the sensible area of the detector $\theta(x)=0$ if $x<0$, $\theta(x)=1$ if $x \geqslant 0 . \hat{J}_{a}$ is the current associated with the measured quantity, such that the output of the detector does not depend on the time each particle takes to cross its sensible area. Indeed, the equation of motion for the "measuring" operator is

$$
\frac{d \hat{K}_{a}(t)}{d t}=\alpha_{a}[\mathbf{x}(t)] \lambda \hat{J}_{a}(t) .
$$

In the equation above, we have assumed that the operator $\hat{K}_{a}$ commutes with the unperturbed Hamiltonian of the detector. In general, however, $\left\langle\hat{K}_{a}\right\rangle$ will fluctuate in time due to background noise. Such fluctuations put a lower limit to the resolution of the detector. For a reliable detection, the resolution must be smaller than the minimal variation $K_{Q a}$ one intends to measure.

Let us introduce proper units. We consider the case where the measured quantities have discrete values proportional to a quantum $E_{Q a}$. For instance, for charge $E_{Q}=e$, the elementary charge, and for spin $E_{Q}=\hbar / 2$. Every time an elementary unit passes the detector, the readout of the latter will change by $K_{Q a}=\lambda_{a} E_{Q a}$. Thus, we introduce the number and phase operators $N_{a}=K_{a} / K_{Q a}, \phi_{a}=V / V_{Q a}$, with $V_{Q a}=\hbar / K_{Q a}$. We further assume that (i) the detectors are initially prepared in a state with zero counts $\hat{\rho}_{\text {det }}(0)=|N=0\rangle\langle N=0|$ and (ii) the spread of the system wave packet is much smaller than the distance between two subsequent detectors $\Delta x \ll X_{a+1}-X_{a}$. The first assumption implies that

$$
\rho_{\mathrm{det}}^{\phi, \phi^{\prime}}(\tau)=\mathcal{Z}\left(\phi, \phi^{\prime}\right) \equiv \int \frac{d \mu}{2 \pi} \frac{d \mu^{\prime}}{2 \pi} \mathcal{Z}_{\mu, \mu^{\prime}}^{\phi, \phi^{\prime}}
$$

or, explicitly,

$$
\begin{aligned}
\mathcal{Z}\left(\phi^{+}, \phi^{-}\right)= & \int \frac{d \mu^{+}}{2 \pi} \frac{d \mu^{-}}{2 \pi} \int_{\mu^{+}}^{\phi^{+}} \mathcal{D} \phi^{+}(t) \int_{\mu^{-}}^{\phi^{-}} \mathcal{D} \phi^{-}(t) \\
& \times \exp \left(S_{\mathrm{det}}\left[\phi^{+}\right]-S_{\mathrm{det}}\left[\phi^{-}\right]+\mathcal{F}_{\mathrm{sys}}\left[\phi^{+}, \phi^{-}\right]\right),
\end{aligned}
$$

where the limits of the path integrals fix the values of the fields at $t=0$ and $t=\tau$, and we introduced the influence functional of the system on the detectors ${ }^{22}$

$$
\begin{aligned}
\exp \left(\mathcal{F}_{\text {sys }}\left[\phi^{+}, \phi^{-}\right]\right):= & \operatorname{Tr}_{\text {sys }}\left\{\exp \left(S_{\text {int }}\left[\phi^{+}, \hat{J}\right]\right) \hat{\rho}_{\text {sys }}(0)\right. \\
& \left.\times \exp \left(-S_{\text {int }}\left[\phi^{-}, \hat{J}\right]\right)\right\},
\end{aligned}
$$

where $S_{\text {int }}$ is the action corresponding to the interaction $H_{\text {int }}$ given in Eq. (3). We shall call $\mathcal{Z}$ the quantum generating function. In principle it depends on twice as many parameters than the classical generating function does. In the rest of the paper we shall use the cumulant generating function (CGF), $\mathcal{F} \equiv \ln \mathcal{Z}$. The advantage of working with the CGF is that it often has a clearer interpretation than $P_{\tau}$, since independent processes contribute factors to $P_{\tau}$ and simply additive terms to the CGF. Hence, if subsequent events are independent, the CGF is proportional to the observation time $\tau$. Thus, time averaged cumulants, which for long $\tau$ correspond to zero-frequency noise and higher order correlators for currents, have a finite value.

\section{DETECTORS WITH NO DYNAMICS}

We analyze the situation where the dynamics of the detectors is neglected. This means that

$$
\exp S_{\operatorname{det}}[\phi(t)]=\prod_{t} \delta[\phi(t)-\phi],
$$

i.e., the counting fields are constant.

We consider first the case of one detector. Then $\mathcal{Z}_{\mu^{+}, \mu^{-}}^{\phi^{+}, \phi^{-}}$ $=\delta_{\phi^{+}, \mu^{+}} \delta_{\phi^{-}, \mu^{-}} \mathcal{Z}\left(\phi^{+}, \phi^{-}\right)$, and

$$
\mathcal{Z}^{\phi^{+}, \phi^{-}}=\operatorname{Tr}_{\text {sys }}\left\{\mathcal{U}^{\phi^{+}} \hat{\rho}_{\text {sys }}(0) \mathcal{U}^{\phi^{-\dagger}}\right\},
$$

where

$$
\mathcal{U}^{\phi}=\mathcal{T} \exp \left[-i \phi \int d t \hat{J}(t) / E_{Q}\right]
$$

( $\mathcal{T}$ being the time-ordering operator) is an operator in the system's Hilbert space. By exploiting the cyclic property of the trace, we have that, if $\hat{J}$ is a conserved operator or, more generally, $\left[\hat{J}(t), \hat{J}\left(t^{\prime}\right)\right]=0$, then $\mathcal{Z}\left(\phi^{+}, \phi^{-}\right)$depends only on $\phi=\phi^{+}-\phi^{-}$. It has been shown that in this case $\mathcal{Z}\left(\phi^{+}, \phi^{-}\right)$ gives directly the generating function. ${ }^{15}$

Next, we consider the case of two detectors. The kernel $\mathcal{Z}$ is now

$$
\mathcal{Z}\left(\phi^{+}, \phi^{-}\right)=\operatorname{Tr}_{\text {sys }}\left\{\mathcal{U}_{2}^{+} \mathcal{U}^{\phi_{1}^{+}} \hat{\rho}_{\text {sys }}(0) \mathcal{U}^{\phi_{1}^{-\dagger}} \mathcal{U}^{\phi_{2}^{-\dagger}}\right\}
$$

Here we exploited assumption (ii) and defined

$$
\mathcal{U}^{\phi_{a}}=\mathcal{T} \exp \left[-i \phi_{a} \int d t \hat{J}_{a}(t) / E_{Q}\right] .
$$

Once again we exploit the cyclic property of the trace and see that the expression does not depend on the combination $\Phi_{2} \equiv \phi_{2}^{+}+\phi_{2}^{-}$. From Eq. (7) we see that in general, for two detectors, $\mathcal{Z}$ does depend on $\Phi_{1}$, even when $\hat{J}_{a}$ are conserved. However, when the system is initially in the unpolarized state $\hat{\rho}_{\text {sys }} \propto \mathcal{I}_{\text {sys }}$, the dependence on $\Phi_{1}$ disappears as well. Another case in which this happens is when the detectors monitor two commuting degrees of freedom which are 
conserved. For instance, if the current $\hat{J}$ is not conserved, in general $\left[\hat{J}(t), \hat{J}\left(t^{\prime}\right)\right] \neq 0$. Thus, even if one repeats the same measurement, one would obtain different results. If, however, the current is conserved $\hat{J}(t)=\hat{J}$, and both detectors measure $\hat{J}$, the kernel depends only on the combination $\phi_{1}$ $+\phi_{2}$, which means that the two measurements will give the same result.

In general, when there are three detectors, labeled 1, 2 and 3 according to their order, measuring noncommuting quantities, even if the system is initially unpolarized, the integrand will depend on the classical variable of the middle detector $\Phi_{2}$. When such a dependence appears in the expression for the generating function, it is a signal that the internal dynamics of the detector must be taken into account. Indeed, when $\mathcal{Z}$ does not depend on $\Phi$, the density matrix is diagonal in the basis $|N\rangle$. When $\mathcal{Z}$ does depend on $\Phi, \rho_{\text {det }}$ develops offdiagonal components. We consider as an example the case where the detectors' density matrix is prepared in a diagonal state at $t=0$, and two particles are sent to the detectors one at time $t_{1}>0$ and the other at time $t_{2}>t_{1}$, in such a way that their wave packets do not overlap. Then, after the first particle has crossed the detectors, the density matrix of the detectors $\rho_{\mathrm{det}}^{N, N^{\prime}}$ has off-diagonal elements, which depend on the original diagonal elements (probabilities). Since one observes only the probabilities, this cannot be ascertained directly. However, when the second particle crosses the detectors, the new probabilities will be a combination of the former diagonal and off-diagonal elements. In order to know $\rho_{\operatorname{det}}^{N, N}\left(t_{2}\right)$, knowledge of $\rho_{\operatorname{det}}^{N, N}\left(t_{1}\right)$ is not sufficient. Thus, the process is non-Markovian. In principle, even after the detector has been measuring for a long time a large number of particles, the off-diagonal elements created after the passage of the first particle will still influence its dynamics. This is not realistic, since, because of the coupling of the detectors to the environment, the off-diagonal elements will go to zero within a typical time $\tau_{c}$. In order to account for this, one should consider the dynamics of the detectors, which we shall do in the next section.

\section{DETECTORS WITH INTERNAL DYNAMICS}

We model the decoherence of the detectors by introducing a dissipative dynamics for the detectors' degrees of freedom, i.e., we couple the detectors to an environment, whose degrees of freedom are traced out. We model the environment as a system of independent harmonic oscillator in thermal equilibrium, having the action

$$
S_{\mathrm{env}}=-\frac{i}{\hbar} \int d t \sum_{j} \frac{1}{2} m_{j}\left[\dot{x}_{j}^{2}-\omega_{j}^{2} x_{j}^{2}\right],
$$

and coupling to the detectors through the position operator

$$
S_{\text {det-env }}=\frac{i}{\hbar} \int d t \sum_{j a} c_{j a} x_{j} V_{Q a} \phi_{a},
$$

with $c_{j a}$ coupling constant between the $j$ th oscillator and the $a$ th detector. Then the generating function becomes

$$
\begin{aligned}
\mathcal{Z}\left(\phi^{+}, \phi^{-}\right) & \\
= & \int \frac{d \mu^{+}}{2 \pi} \frac{d \mu^{-}}{2 \pi} \int d x_{j} d x_{j}^{+} d x_{j}^{-} \int_{\mu^{+}}^{\phi^{+}} \mathcal{D} \phi^{+}(t) \int_{\mu^{-}}^{\phi^{-}} \mathcal{D} \phi^{-}(t) \\
& \times \int_{x_{j}^{+}}^{x_{j}} \mathcal{D} x_{j}^{+}(t) \int_{x_{j}^{-}}^{x_{j}} \mathcal{D} x_{j}^{-}(t) \rho_{\mathrm{env}}\left(x^{+}, x^{-}\right) \\
& \times \exp \left\{S_{\mathrm{det}}\left[\phi^{+}\right]-S_{\mathrm{det}}\left[\phi^{-}\right]+F_{\mathrm{sys}}\left[\phi^{+}, \phi^{-}\right]\right. \\
& +S_{\text {env }}\left[x_{j}^{+}\right]-S_{\mathrm{env}}\left[x_{j}^{-}\right]+S_{\mathrm{det}-\mathrm{env}}\left[x_{j}^{+}, \phi^{+}\right] \\
& \left.-S_{\text {det-env }}\left[x_{j}^{-}, \phi^{-}\right]\right\},
\end{aligned}
$$

In the expression above, we isolate the part

$$
\begin{aligned}
\exp \mathcal{F}_{\text {env }}= & \int d x_{j} d x_{j}^{+} d x_{j}^{-} \int_{x_{j}^{+}}^{x_{j}} \mathcal{D} x_{j}^{+}(t) \int_{x_{j}^{-}}^{x_{j}} \mathcal{D} x_{j}^{-}(t) \\
& \times \exp \left(S_{\text {env }}\left[x_{j}^{+}\right]-S_{\text {env }}\left[x_{j}^{-}\right]+S_{\text {det-env }}\left[x_{j}^{+}, \phi^{+}\right]\right. \\
& \left.-S_{\text {det-env }}\left[x_{j}^{-}, \phi^{-}\right]\right) \rho_{\text {env }}\left(x^{+}, x^{-}\right)
\end{aligned}
$$

which gives the influence functional of the environment on the detectors. We notice from Eq. (8) that, since the functions $\phi_{a}^{ \pm}(t)$ are fixed by the external path integrals, they act as an external source $I_{j}^{ \pm}(t)=\Sigma_{a} c_{j a} \phi_{a}^{ \pm}(t)$ on the $j$ th harmonic oscillator. It is then possible to perform the independent Gaussian path integrals over $x_{j}$, resulting in ${ }^{23}$

$$
\begin{aligned}
\mathcal{F}_{\text {env }}= & -\frac{i}{\hbar} \sum_{a} V_{Q a}^{2} \int_{0}^{\tau} d t \int_{0}^{t} d t^{\prime}\left[\phi_{a}^{+}(t)-\phi_{a}^{-}(t)\right] \\
& \times\left[\alpha_{a}\left(t-t^{\prime}\right) \phi_{a}^{+}\left(t^{\prime}\right)-\alpha_{a}^{*}\left(t-t^{\prime}\right) \phi_{a}^{-}\left(t^{\prime}\right)\right],
\end{aligned}
$$

where the influence of the environment is contained in the complex functions $\alpha_{a}(t)$, whose Fourier transforms are

$$
\alpha_{a}(\omega)=\frac{1}{2}\left(\operatorname{coth} \frac{\hbar \beta \omega}{2}+1\right) \sigma_{a}(\omega),
$$

where the inverse temperature $\beta=1 / k_{B} T$ comes from having assumed the bath in thermal equilibrium $\left[\hat{\rho}_{\text {env }}\right.$ $\left.=\exp \left(-\beta \hat{H}_{\text {env }}\right)\right]$, and $\sigma_{a}$ are the spectral densities

$$
\sigma_{a}(\omega)=\pi \sum_{j} \frac{c_{j a}^{2}}{m_{j} \omega_{j}}\left[\delta\left(\omega-\omega_{j}\right)-\delta\left(\omega+\omega_{j}\right)\right] .
$$

At low frequencies, we can approximate the odd functions $\sigma_{a}$ by $\sigma_{a}(\omega) \simeq \gamma_{a} \omega$ (Ohmic approximation), with $\gamma_{a}$ friction constant, as will be clear later. We introduce new variables $\phi=\phi^{+}-\phi^{-}, \Phi=\phi^{+}+\phi^{-}$. Thus we get

$$
\begin{aligned}
\mathcal{F}_{\text {env }}= & \sum_{a} \gamma_{a} V_{Q a}^{2}\left\{\frac{1}{2 \hbar} \int \frac{d \omega}{2 \pi} \omega \Phi_{a}(\omega) \phi_{a}(-\omega)\right. \\
& \left.-\frac{1}{\beta \hbar^{2}} \int \frac{d \omega}{2 \pi} \frac{\beta \hbar \omega}{2} \operatorname{coth} \frac{\beta \hbar \omega}{2}\left|\phi_{a}(\omega)\right|^{2}\right\} .
\end{aligned}
$$

We take the action of free detectors to be that of harmonic oscillators, i.e., 


$$
\mathcal{S}_{\mathrm{det}}[\phi]=\sum_{a} \frac{-i m_{a} V_{Q a}^{2}}{2 \hbar} \int \frac{d \omega}{2 \pi}\left(\omega^{2}-\Omega_{a}^{2}\right)\left|\phi_{a}(\omega)\right|^{2},
$$

where $m_{a}$ is the "mass" of the detector (i.e., it is the inertial term corresponding to the kinetic energy $\left.m_{a} V_{Q a}^{2} \dot{\phi}_{a}^{2} / 2\right)$. Then the generating function reads

$$
\begin{aligned}
\mathcal{Z}(\phi, \Phi)= & \int \frac{d \mu}{2 \pi} \frac{d M}{2 \pi} \int_{\mu}^{\phi} \mathcal{D} \phi(t) \int_{M}^{\Phi} \mathcal{D} \Phi(t) \\
& \times \exp \left\{\sum _ { a } \left[\frac{-i m_{a} V_{Q a}^{2}}{2 \hbar} \int \frac{d \omega}{2 \pi} \Phi_{a}(\omega) g_{a}^{-1}(\omega)\right.\right. \\
& \left.\times \phi_{a}(-\omega)-\frac{\gamma_{a}}{\beta \hbar^{2}} \int \frac{d \omega}{2 \pi} f(\omega)\left|\phi_{a}(\omega)\right|^{2}\right] \\
& \left.+\mathcal{F}_{\text {sys }}[\phi, \Phi]\right\},
\end{aligned}
$$

where we introduced the response function

$$
g_{a}^{-1}(\omega)=\omega^{2}-\Omega_{a}^{2}+i \frac{\gamma_{a}}{m_{a}} \omega,
$$

from which one can see that $\gamma_{a}$ are proportional to the friction constant, and the fluctuation term

$$
f(\omega)=\frac{\beta \hbar \omega}{2} \operatorname{coth} \frac{\beta \hbar \omega}{2} .
$$

The part of the action containing the fluctuation term in $\phi(\omega)$ is, at low frequencies, proportional to temperature $T$ and to $1 / \hbar^{2}$. The factor $1 / \hbar^{2}$ strongly suppresses large fluctuations in $\phi$. Thus, the influence functional due to the measured system $\mathcal{F}_{\text {sys }}[\Phi, \phi]=\int d t L_{\text {inf }}(\Phi(t), \phi(t))$ can be approximated by $\int d t L_{\text {inf }}(\Phi(t), \phi):=\mathcal{F}_{\phi}[\Phi]$. Integration over $\phi_{\omega}$ gives finally

$$
\mathcal{Z}(\phi, \Phi)=\int d M \int_{M}^{\Phi} \mathcal{D} \Phi(t) e^{\mathcal{F}_{\phi}[\Phi]+S_{\text {eff }}[\Phi]},
$$

with the effective action

$$
S_{\mathrm{eff}}[\Phi]=-\frac{1}{2} \sum_{a} \frac{\left(\beta m_{a} V_{Q a}^{2}\right)^{2}}{\gamma_{a}} \int d \omega \frac{\left|g_{a}^{-1}(\omega)\right|^{2}}{f(\omega)}\left|\Phi_{a}(\omega)\right|^{2} .
$$

We notice that at high temperatures $f(\omega) \simeq 1$, and thus $\hbar$ disappears in the effective action for $\Phi$. For this reason the latter is termed the "classical" part of the field.

In the limit of small mass $m_{a} \rightarrow 0, m_{a} \Omega_{a}^{2} V_{Q a}^{2} \rightarrow E_{a}$, where $E_{a}$ has a finite value and is a typical energy scale of detector $a$, the effective action simplifies to

$$
S_{\mathrm{eff}}[\Phi]=-\frac{1}{2} \sum_{a} \int d t\left[\tau_{a c}\left[\dot{\Phi}_{a}(t)\right]^{2}+\frac{1}{\tau_{a c} \Delta \Phi_{a}^{2}} \Phi_{a}(t)^{2}\right],
$$

with $\tau_{a c}=\beta \gamma_{a} V_{Q a}^{2} / 2$ the "coherence time" of the detector, and $\Delta \Phi_{a}=2 / \beta E_{a}$ the spread of $\Phi_{a}$.

\section{SPIN DETECTOR}

We discuss a model for spin detection. The setup corresponds to the one proposed and used in Ref. 24 to detect

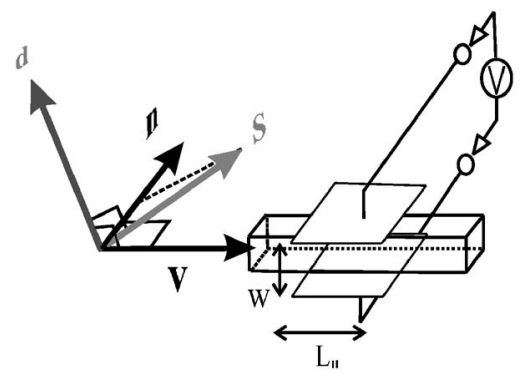

FIG. 1. The proposed spin current detector. An electron with velocity $\mathbf{v}$ and spin $\mathbf{S}$ induces a voltage drop in a capacitor. The electric field $\mathbf{E}$ inside the capacitor produces an Aharonov-Casher phase shift on the electrons.

Aharonov-Casher effect ${ }^{25}$ for neutrons. This setup exploits the fact that a moving magnetic dipole generates an electric one. ${ }^{26,27}$ To measure this, one encloses the two-dimensional current lead between the plates of a capacitor as shown in Fig. 1. While in Ref. 24 the neutrons passed a fixed electric field, which gave a constant Aharonov-Casher phase, in a spin detector the initial voltage applied to the plates is zero, and the passing of a particle with spin $1 / 2$ will cause the charge in the capacitor to show pulses towards positive or negative values depending on the result of the measurement. The associated phase $K_{t}=\int_{0}^{t} d t Q(t)$ will thus increase or decrease stepwise in the ideal situation where spins are transmitted separately in vacuum through the detector.

Each spin moving with velocity $\mathbf{v}$ produces an electric field. For electrons in vacuum, the interaction term between spin and detector is given by the spin-orbit coupling

$$
H_{\text {int }}=-\frac{1}{2} \mathbf{E} \cdot\left(\frac{\mathbf{v}}{c^{2}} \times \mu\right),
$$

with $c$ the speed of light, and the factor $1 / 2$ accounts for the Thomas precession. The magnetic moment $\mu$ is proportional to the spin $\mu=\left(g_{S}|e| / 2 m_{e}\right) S$, with $m_{e}$ mass of the electron, $e=-|e|$ its charge, and $g_{S}$ its spin gyromagnetic factor. Thus, we rewrite the interaction as

$$
H_{\text {int }}=-\left(g_{S}|e| / 4 m_{e} c^{2}\right) \mathbf{E} \cdot(\mathbf{v} \times \mathbf{S}) .
$$

The spin-orbit coupling induces a current in the $R C$ circuit. The integrated charge traversing the circuit is the detector read out. The read-out signal is proportional to spin current in the lead $\mathbf{J}, Q=\lambda \mathbf{n} \cdot \mathbf{J}, \mathbf{n}$ being the unit vector perpendicular to the direction of the current flow and parallel to the plates of the capacitor, $\lambda$ being a proportionality coefficient. The concrete expression for the latter, $\lambda=g_{S}|e| L_{\|} / 4 m_{e} c^{2} w$, depends on the geometrical dimensions of the detector the length of its plates in the direction of the current $L_{\|}$, and the distance between the plates $w$. The variable canonically conjugated to the read out is the voltage $V$ across the capacitor, and the expression for the interaction in terms of $V$ contains the same proportionality coefficient $\lambda, H_{\text {int }}=-\lambda V \mathbf{n} \cdot \mathbf{J}$. Our choice of the detection setup is motivated by the fact that this detector does not influence electron transfers through the contact and only gives the minimal feedback compatible with the uncertainty principle: the electrons passing the ca- 


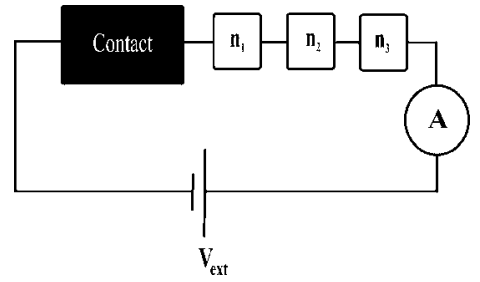

FIG. 2. The setup considered, in the case of three spin detectors and one charge detector.

pacitor in the direction of current acquire an AharonovCasher phase shift, which consists in a precession of the spin around the detection axis $\mathbf{n}$. This depends on spin and is given by $\Phi_{\mathrm{ac}}=\lambda V \mathbf{n} \cdot \mathbf{S} / \hbar$. This is similar to the detection scheme presented in Ref. 2 for charges transferred. A fundamental complication in comparison with the charge FCS is that in our case the phase shift depends on spin, so that even the minimal feedback influences the statistics of the outcomes of following spin detectors. We introduce dimensionless variables $N=2 \int d t Q / \hbar \lambda, \phi=\lambda V / 2$. Then $N$ varies by one every time a spin $1 / 2$ crosses the detector. With reference to Eqs. (17) and (18), we have $m=L C^{2} \rightarrow 0, \Omega^{2}$ $=1 / L C \rightarrow \infty, m \Omega^{2} \rightarrow C, \gamma \rightarrow R C^{2}, E=4 C / \lambda^{2}$, with $L, R$, and $C$ inductance (assumed negligible), resistance and capacitance of the circuit.

\section{THE SETUP CONSIDERED}

We consider a system composed of two metallic, unpolarized leads, connected through a coherent conductor, characterized by a set of transmission probabilities $T_{n}$, where $n$ identifies transmission channels. A negative bias voltage $V$ is applied to the left lead. At the right of the conductor there are several spin detectors, labeled from left to right by $a$ $=1,2 \ldots$, and a current detector, denoted by $a=0$. The counting fields will be then $\phi_{a}$, with $a=0,1, \ldots$. Since charge and spin currents commute, the current detector can be positioned at any point along the chain of detectors, without influencing the statistics of the outcomes. We assume that the distance of the last detector from the scattering region (the coherent conductor) is such that the average time for an electron to go from the scattering region to the detector is much smaller than the spin decoherence time. In Ref. 28, indeed, it was proved that the third cumulant for charge transfer depends on the position of the detector.

The setup is depicted in Fig. 2. We require that the coherent conductor is nonpolarizing. Thus, the average spin current is zero. However, there are spin fluctuations, which are revealed by measuring noise and higher order correlators (or cumulants). From the symmetry with respect to reversal of spin, we can predict $a$ priori that all odd cumulants are zero. We shall concentrate on a situation where there are three spin detectors. This is because, as anticipated in Sec. II, the current is unpolarized and one needs at least three detectors monitoring noncommuting quantities in order to see nontrivial consequences of the detectors' feedback on the system. The feedback consists in the wave function picking up an Aharonov-Casher phase while traversing each detector.

\section{RESULTS}

The technique we use is an extension of the scattering theory of charge FCS. This theory ${ }^{2,3,6}$ expresses FCS in terms of a phase factor $e^{i \chi}$ acquired by scattering waves upon traversing the charge detector.

Since we do not consider energy-resolved measurements, the phase factor does not depend on the channel, and the approach works for a multichannel conductor as well as for a single-channel one. The phase factor $e^{i \chi}$ can be seen as resulting from a gauge transform, to be applied to the (known) Green function of the right lead, that removes the coupling term $^{2,6} \hat{H}_{\mathrm{int}}=-(\hbar / e) \hat{\chi} \hat{I}$. For the case of the spin detectors, the gauge transform introduces a phase factor which is a unitary matrix in spin space. Namely, the gauge transform generated by spin detector $a$ is $e^{i \phi_{a} \mathbf{n}_{a} \tau}$. In this matrix, $\boldsymbol{\tau}$ is a pseudovector of $2 \times 2$ Pauli matrices, and $\mathbf{n}_{a}$ is the direction along which detector $a$ detects spin current. The Keldysh Green function of the lead is

$$
\check{G}_{l}(E)=\left(\begin{array}{cc}
1-2 f_{l} & -2 f_{l} \\
-2\left(1-f_{l}\right) & 2 f_{l}-1
\end{array}\right),
$$

where $l \in\{L, R\}$ denotes the left or right lead, and $f_{l}$ is the corresponding Fermi occupation number at energy $E$ and chemical potential $\mu_{l}$. The elements of the matrix are actually in their turn a matrix in spin space. Since the leads are assumed to be unpolarized, they are simply the identity. The matrix current is given by ${ }^{7}$

$$
\check{I}(\chi, \phi)=\frac{e^{2}}{2 \pi \hbar} \sum_{n} \frac{T_{n}\left[\check{G}_{L}, \check{\widetilde{G}}_{R}\right]}{1+T_{n}\left(\left\{\check{G}_{L}, \check{\tilde{G}}_{R}\right\}-2\right) / 4},
$$

from which it follows that the quantum generating function is

$$
\mathcal{F}=\frac{e^{2}}{2 \pi \hbar} \sum_{n} \int d E \ln \left[1+T_{n}\left(\left\{\check{G}_{L}, \check{\widetilde{G}}_{R}\right\}-2\right) / 4\right]
$$

Here $[\cdots](\{\cdots\})$ denote (anti)commutator of two matrices, and $\check{\widetilde{G}}_{R}$ is the transformed matrix

$$
\check{\widetilde{G}}_{R}=e^{i \bar{\chi}} \prod_{a} e^{i \bar{\phi}_{a} \mathbf{n}_{a} \cdot \check{G}_{R}} \varlimsup_{a}^{\leftarrow} e^{-i \bar{\phi}_{a} \mathbf{n}_{a} \cdot \tau} e^{-i \bar{\chi}},
$$

where $\bar{\chi}=\operatorname{diag}\left(\chi^{+}, \chi^{-}\right), \quad \bar{\phi}=\operatorname{diag}\left(\phi^{+}, \phi^{-}\right)$are matrices in Keldysh space. After substituting the expression for $\check{G}_{R}$, we obtain

$$
\check{\widetilde{G}}_{R}=\left(\begin{array}{cc}
1-2 f_{R} & -2 f_{R} e^{i \chi} \mathcal{M} \\
-2\left(1-f_{R}\right) e^{-i \chi} \mathcal{M}^{\dagger} & 2 f_{R}-1
\end{array}\right),
$$

where

$$
\mathcal{M} \equiv \prod_{a}^{\rightarrow} e^{i\left(\phi_{a}^{+} / 2\right) \mathbf{n}_{a} \cdot \tau} \prod_{a}^{\leftarrow} e^{-i\left(\phi_{a}^{-} / 2\right) \mathbf{n}_{a} \cdot \tau}
$$

is a matrix in spin space. We notice that (i) the charge fields come only in the combination $\chi=\chi^{+}-\chi^{-}$and (ii) the phase 
factors $e^{i \chi}$ in the expression for charge FCS are replaced by $e^{i \chi} \mathcal{M}$ to give the FCS of charge and spin counts after taking trace over spin. If we also notice that $\mathcal{M}$ is a $(2 \times 2)$ matrix with eigenvalues $e^{ \pm i \alpha}$, we arrive at

$$
\mathcal{F}\left(\chi,\left\{\phi_{a}^{+}\right\},\left\{\phi_{a}^{-}\right\}\right)=\frac{1}{2} \sum_{ \pm} \mathcal{F}_{c}(\chi \pm \alpha),
$$

where $\mathcal{F}_{c}(\chi)$ is the generating function for charge counting. The $\alpha$ is given by

$$
\cos \alpha=\frac{1}{2} \operatorname{tr} \mathcal{M} .
$$

The explicit expression for the system considered here, in terms of the transmission probabilities through the contact and the applied bias is, at zero temperature,

$$
\mathcal{F}=\int_{0}^{\tau} \frac{d t}{\tau_{V}} \sum_{n} \ln \left[R_{n}^{2}+T_{n}^{2} e^{2 i \chi}+2 R_{n} T_{n} e^{i \chi} \cos \alpha\right],
$$

with $R_{n} \equiv 1-T_{n}, \tau_{V} \equiv 2 \pi \hbar / \mathrm{eV}$. The interpretation is quite straightforward: electrons coming through different channels behave independently, which is revealed by the fact that the generating function splits into a sum; each channel can accommodate two electrons in a spin-singlet configuration; with probability $R_{n}^{2}$ none of the two electrons passes the junction, and there is no contribution to the charge counting nor to the spin one; with probability $T_{n}^{2}$ both electrons come through the conductor. This gives a contribution of two elementary charges transferred (factor $e^{2 i \chi}$ ), but no spin transfer. Finally, with probability $p_{n}=2 R_{n} T_{n}$, exactly one of the two electrons is transferred. This gives a contribution to the charge and to the spin counting.

\section{PROJECTION POSTULATE}

We demonstrate that a different FCS is predicted by using a different approach, namely, a naive application of the projection postulate, consisting in avoiding the description of the measurement and applying the projection to the system measured. We shall denote this procedure with PP for brevity. This approach predicts a parameter $\alpha_{\mathrm{PP}}$ which does not depend on $\Phi$. Let us give the details of such a derivation: When an unpolarized electron arrives to the first detector, the probability of the outcome $\sigma_{1}= \pm 1$ is $P_{1}\left(\sigma_{1}\right)=1 / 2$. The conditional probability that the second detector gives $\sigma_{2}$, given that the first read $\sigma_{1}$ is $P_{2}\left(\sigma_{2} \mid \sigma_{1}\right)=\left(1+\sigma_{1} \sigma_{2} \mathbf{n}_{1} \cdot \mathbf{n}_{2}\right) / 2$. This is because after the first detection the spin of the electron is assumed to have collapsed along $\pm \mathbf{n}_{1}$. The same happens after the second detection. Consequently, the conditional probability that a third detector reads $\sigma_{3}$, given that the first read $\sigma_{1}$ and the second $\sigma_{2}$, depends only on the latter outcome $P_{3}\left(\sigma_{3} \mid \sigma_{2}, \sigma_{1}\right)=\left(1+\sigma_{2} \sigma_{3} \mathbf{n}_{2} \cdot \mathbf{n}_{3}\right) / 2$. The process is in a sense a Markovian one. The total joint probability for each electron transfer with an arbitrarily long chain of detectors is

$$
P(\{\sigma\})=\frac{1}{2} \prod_{a=1}^{K-1} \frac{1+\sigma_{a} \sigma_{a+1} \mathbf{n}_{a} \cdot \mathbf{n}_{a+1}}{2},
$$

and the corresponding generating function for the setup considered here is given by Eq. (24) with

$$
\cos \alpha_{\mathrm{PP}}=\sum_{\{\sigma\}} \cos \left[\sum_{a} \sigma_{a} \phi_{a}\right] \frac{1}{2} \prod_{a=1}^{K-1} \frac{1+\sigma_{a} \sigma_{a+1} \mathbf{n}_{a} \cdot \mathbf{n}_{a+1}}{2} .
$$

\section{COMPARISON OF THE TWO APPROACHES FOR ONE AND TWO SPIN DETECTORS}

Now, let us go back to Eqs. (21) and (23) and compare the two approaches for some simple cases. For the case of one or two detectors in series, the eigenvalues $e^{ \pm i \alpha}$ are not affected by the order of matrix multiplication in Eq. (21) and depend on differences of spin counting fields $\phi_{a} \equiv \phi_{a}^{+}-\phi_{a}^{-}$only (in fact they coincide with the value $e^{\left. \pm i \alpha_{\mathrm{PP}}\right)}$. This implies that the FCS definition (4) can be readily interpreted in classical terms: it is a generating function for probability distribution of a certain number of spin counts $\sigma_{a}$ in each detector,

$$
P\left(\left\{\sigma_{a}\right\}\right)=\int \prod_{a} d \phi_{a} e^{F\left(0,\left\{\phi_{a}\right\}\right)} e^{-i \Sigma_{a} \sigma_{a} \phi_{a}} .
$$

For a single detector, the spin FCS is very simple: it corresponds to independent transfers of two sorts of electrons, with spins "up" and "down" with respect to the quantization axis. The cumulants of the spin (charge) transferred are given by the derivatives of $F$ with respect to $\phi_{1}(\chi)$, at $\chi=\phi_{1}=0$. In this case $\alpha=\phi_{1}$. From this and relation (22), we conclude that all odd cumulants of spin current are 0 , as anticipated, and all even cumulants coincide with the charge cumulants.

For two spin detectors, with $\mathbf{n}_{1} \cdot \mathbf{n}_{2}=\cos \theta$, we obtain $\cos \alpha=\cos \phi_{1} \cos \phi_{2}-\sin \phi_{1} \sin \phi_{2} \cos \theta$. Since there is no dependence on $\Phi_{a}$, the quantum generating function has an immediate interpretation; we consider the case when the read out of the charge is not exploited $(\chi=0)$. Then

$$
\mathcal{Z}(\phi)=\prod_{n}\left[q_{n}+p_{n} \cos \alpha\right]^{M},
$$

where $p_{n}=2 R_{n} T_{n}$ is the probability that, in two attempts of transmitting one electron over a spin degenerate channel $n$, exactly one is transmitted and $q_{n}=1-p_{n}$. This result coincides with what one would obtain from the projection postulate.

We discuss in detail the probability distribution. By performing the Fourier transform, we find the probability of detecting a spin $\sigma_{1}$ in direction $\mathbf{n}_{1}$ and $\sigma_{2}$ in direction $\mathbf{n}_{2}$ :

$$
P\left(\sigma_{1}, \sigma_{2}\right)=\sum_{\sigma_{1}^{(n)}} \sum_{\sigma_{2}^{(n)}} \prod_{n} P_{n}\left(\sigma_{1}^{(n)}, \sigma_{2}^{(n)}\right),
$$

where the prime in the sum means that it is restricted to $\Sigma_{n} \sigma_{a}^{(n)}=\sigma_{a}$, and the probability for each channel $n$ is 


$$
\begin{aligned}
P_{n}\left(\sigma_{1}, \sigma_{2}\right)= & \sum_{k} P_{\mathrm{tr}}(k \mid N) P_{\uparrow}\left[\left(k+\sigma_{1}\right) / 2 \mid k\right] \\
& \times \sum_{l} P_{\uparrow}\left[\left(k+\sigma_{2}+2 l\right) / 4 \mid\left(k+\sigma_{1}\right) / 2=\uparrow\right] \\
& \times P_{\uparrow}\left[\left(k+\sigma_{2}-2 l\right) / 4 \mid\left(k-\sigma_{1}\right) / 2=\downarrow\right],
\end{aligned}
$$

where

$$
\begin{gathered}
P_{\mathrm{tr}}(k \mid N)=\left(\begin{array}{c}
N \\
k
\end{array}\right) p_{n}^{k} q_{n}^{N-k}, \\
P_{\uparrow}(l \mid k)=\frac{1}{2^{k}}\left(\begin{array}{c}
k \\
l
\end{array}\right), \\
P_{\uparrow}(l \mid k=\uparrow)=\left(\begin{array}{l}
k \\
l
\end{array}\right)\left[\cos ^{2}(\theta / 2)\right]\left[\sin ^{2}(\theta / 2)\right]^{k-l}, \\
P_{\uparrow}(l \mid k=\downarrow)=\left(\begin{array}{l}
k \\
l
\end{array}\right)\left[\cos ^{2}(\theta / 2)\right]^{k-l}\left[\sin ^{2}(\theta / 2)\right]^{l} .
\end{gathered}
$$

The sums are over all values for which the binomials make sense (no negative nor half-integer values). Thus $k, l$, $\sigma_{1}, \sigma_{2}$ have the same parity. $P$ can be interpreted as follows: since the current is unpolarized, we can think of it as carried by pairs of electrons in singlet configuration. Then, there is a successful attempt to transfer spin when exactly one of the two electrons is transmitted. This gives $P_{\text {tr }}(k \mid N)$, the probability of transferring $k$ spins over $N$ attempts ( $p_{n}$ probability of success for a single attempt) through channel $n$; the second binomial comes from the ways one can pick $N_{1 \uparrow}=(k$ $+\sigma_{1}$ )/2 spins up out of $k$ spins, with probability $1 / 2$ (we recall that the incoming electrons are unpolarized); the third term comes from the fact that, given that $N_{1 \uparrow}=\left(k+\sigma_{1}\right) / 2$ spins up according to the first detector are passed to the second one, the latter will measure $\left(k+\sigma_{2}+2 l\right) / 4$ of these as spins up [the probability of agreement between detectors being $\left.p_{a g}=\cos ^{2}(\theta / 2)\right]$, and the rest as spins down; analogously, the latter term comes from the fact that given $N_{1 \downarrow}=(k$ $\left.-\sigma_{1}\right) / 2$ spins down along direction $\mathbf{n}_{1}$ have been detected, $\left(k+\sigma_{2}-2 l\right) / 4$ of them will be detected from the second detector as spins up, while the remaining ones will be detected as down.

When the two detectors have parallel orientation $(\theta=0)$, the second sum in Eq. (29) is nonzero only if $\sigma_{1}=\sigma_{2}$, giving

$$
P\left(\sigma_{1}, \sigma_{2}\right)=\sum_{k} P_{\mathrm{tr}}(k \mid N) P_{\uparrow}\left(\left(k+\sigma_{1}\right) / 2 \mid k\right) \delta_{\sigma_{1}, \sigma_{2}},
$$

i.e., there is perfect correlation, as is to be expected. When the two detectors have orthogonal orientation $(\theta=\pi / 2)$, it is possible to perform analytically the sum over $m$ :

$$
P\left(\sigma_{1}, \sigma_{2}\right)=\sum_{k} P(k \mid N) P_{\uparrow}\left[\left(k+\sigma_{1}\right) / 2 \mid k\right] P_{\uparrow}\left[\left(k+\sigma_{2}\right) / 2 \mid k\right],
$$

i.e., the outcomes are independent, given that $k$ successful spin transfers happened.

\section{COMPARISON OF THE TWO APPROACHES FOR THREE SPIN DETECTORS}

For the case of three detectors, we have $\cos \alpha=\cos \alpha_{\mathrm{PP}}-\sin \theta_{12} \sin \theta_{23} \sin \left(\Phi_{2}-\Phi_{2}^{(0)}\right) \sin \phi_{3} \sin \phi_{1}$,

$\cos \alpha_{\mathrm{PP}}=\cos \phi_{1} \cos \phi_{2} \cos \phi_{3}-\cos \theta_{12} \sin \phi_{1} \sin \phi_{2} \cos \phi_{3}$

$$
\begin{aligned}
& -\cos \theta_{23} \sin \phi_{2} \sin \phi_{3} \cos \phi_{1} \\
& -\cos \theta_{12} \cos \theta_{23} \sin \phi_{3} \sin \phi_{1} \cos \phi_{2} .
\end{aligned}
$$

Here $\theta_{j k}=\arccos \mathbf{n}_{j} \cdot \mathbf{n}_{k}$ are the angles between the polarizations $(\mathbf{n})$ of detectors $j$ and $k$, and $\cos \Phi_{2}^{(0)}=\left(\mathbf{n}_{1} \times \mathbf{n}_{2}\right) \cdot \mathbf{n}_{3} /$ $\sin \theta_{12} \sin \theta_{23}, \quad \sin \Phi_{2}^{(0)}=\left(\mathbf{n}_{1} \times \mathbf{n}_{2}\right) \cdot\left(\mathbf{n}_{2} \times \mathbf{n}_{3}\right) / \sin \theta_{12} \sin \theta_{23}$. As before $\cos \alpha_{\mathrm{PP}}$ is the part corresponding to the projection postulate. We notice that when two consecutive detectors are parallel or antiparallel, then $\alpha_{\mathrm{PP}}=\alpha$. This is because the same measurement is repeated twice, and thus we fall back to the case of two detectors.

In general, however, $\cos \alpha$ depends on $\Phi_{2}$, and thus one needs to account for the dynamics of the second detector in order to get the probability distribution for the spin counts. We recall that the corresponding detector's action is $S\left[\Phi_{2}\right]$ $=\int d t \frac{1}{2}\left[\tau_{c} \dot{\Phi}_{2}(t)^{2}-\Phi_{2}(t)^{2} / \tau_{c}\left\langle\Phi_{2}^{2}\right\rangle\right]$, with $\tau_{c}$ coherence time and $\left\langle\Phi_{2}^{2}\right\rangle$ fluctuations of $\Phi_{2}$.

We have calculated the second cumulants or cross correlators: we found that they differ from the ones obtained by using PP only by small terms. The correlator between first and third detector's readings is

$$
\left\langle\left\langle\sigma_{1} \sigma_{3}\right\rangle\right\rangle=\left\langle\left\langle N^{2}\right\rangle\right\rangle\left[C+\left(\cos \theta_{13}-C\right) e^{-\left\langle\Phi_{2}^{2}\right\rangle / 2}\right],
$$

where $C \equiv \cos \theta_{12} \cos \theta_{23}$, and the first term is the PP result. The second term, as expected, has a typical signature of interference effects: it is suppressed exponentially if the variance of the corresponding Aharonov-Casher phase $\left\langle\Phi_{2}^{2}\right\rangle \gg 1$. Since $\Phi_{\mathrm{ac}}$ is inversely proportional to $\hbar$, this is the classical limit. In this limit, the result coincides with the PP.

However, fourth cumulants show a large deviation from the PP result. Namely,

$$
\left\langle\left\langle\sigma_{1}^{2} \sigma_{3}^{2}\right\rangle\right\rangle=\left\langle\left\langle\sigma_{1}^{2} \sigma_{3}^{2}\right\rangle\right\rangle_{\mathrm{PP}}+8 \frac{\tau_{c}}{\tau} A\left\langle\left\langle N^{2}\right\rangle\right\rangle^{2},
$$

where $A \equiv \sin ^{2} \theta_{12} \sin ^{2} \theta_{23}$, and the PP result is expressed in terms of charge cumulants as

$$
\left\langle\left\langle\sigma_{1}^{2} \sigma_{3}^{2}\right\rangle\right\rangle_{\mathrm{PP}}=\frac{1}{3}\left[\left(1+2 C^{2}\right)\left\langle\left\langle N^{4}\right\rangle\right\rangle+2\left(1-C^{2}\right)\left\langle\left\langle N^{2}\right\rangle\right\rangle\right] .
$$

This deviation results from correlations of $\Phi_{2}$ at time scale $\tau_{c}$. To estimate the result, we notice that the charge cumulants are of the order of $\tau / \tau_{\mathrm{el}}$, $\tau_{\mathrm{el}}$ being the average time between electron transfers. It is easy to fulfill the condition $\tau_{\text {el }} \ll \tau_{c} \ll \tau$, and in this case $\left\langle\left\langle\sigma_{1}^{2} \sigma_{3}^{2}\right\rangle\right\rangle$ is much larger than PP result.

It is interesting to study further the probability distribution which gives rise to such anomalously large fourth-order cumulants. This we shall do in the next section. 


\section{A PARTICULAR CASE}

We discuss for definiteness the case of three detectors oriented along three orthogonal directions forming a righthanded basis. This implies $\Phi_{2}^{(0)}=0$. We concentrate on the joint probability distribution for the outcomes of the first and the third detector, irrespective of the reading of the second detector. We consider the "classical" limit $\left\langle\Phi_{2}^{2}\right\rangle \rightarrow \infty$. Then, the generating function for the probability $P\left(\sigma_{1}, \sigma_{3}\right)$ for counting $\sigma_{1}, \sigma_{3}$ spins in the detectors is

$$
\begin{aligned}
Z\left(\phi_{1}, \phi_{3}\right)= & \int d \Phi_{2, i} d \Phi_{2, f} \int_{\Phi_{2, i}}^{\Phi_{2, f}} \mathcal{D} \Phi_{2}(t) \\
& \times \exp \left\{\int _ { 0 } ^ { \tau } d t \left[-\frac{\tau_{c}}{2} \dot{\Phi}_{2}^{2}\right.\right. \\
& \left.+\frac{1}{\tau_{V}} \sum_{n} \ln \left[q_{n}+p_{n} \cos \alpha\left(\phi, \Phi_{2}\right)\right]\right],
\end{aligned}
$$

where

$$
\begin{gathered}
\cos \alpha\left(\phi, \Phi_{2}\right)=\cos \alpha_{\mathrm{PP}}-\sin \phi_{3} \sin \phi_{1} \cos \Phi_{2} \\
\cos \alpha_{\mathrm{PP}}=\cos \phi_{1} \cos \phi_{3} .
\end{gathered}
$$

We have a path integral over imaginary time. We exploit the quantum-mechanical technique and reexpress the path integral in terms of amplitudes

$$
Z(\phi)=\int d \Phi_{2, i} d \Phi_{2, f}\left\langle\Phi_{2 f} ; t=i \tau, \phi \mid \Phi_{2 i} ; t=0, \phi\right\rangle .
$$

Here the counting fields $\phi$ are parameters, and the time evolution of the variable $\Phi_{2}$ is dictated by $\left|\Phi_{2} ; t, \phi\right\rangle$ $=e^{-i \hat{H}(\phi) t}\left|\Phi_{2} ; 0, \phi\right\rangle$, with the Hamiltonian

$$
\hat{H}(\phi)=-\frac{1}{2 \tau_{c}} \frac{\partial^{2}}{\partial \Phi_{2}^{2}}-\frac{1}{\tau_{V}} \sum_{n} \ln \left[q_{n}+p_{n} \cos \alpha\left(\phi, \Phi_{2}\right)\right] .
$$

Then, for large values of $\tau$, the path integral can be approximated

$$
Z(\phi) \simeq e^{-E_{0}(\phi) \tau}
$$

where $E_{0}(\phi)$ is the ground-state energy of the Hamiltonian.

The next step is to find an explicit expression for the probability. We recall that the probability to have detectors 1 and 3 measure average spin currents $I_{1}=\sigma_{1} / \tau, I_{3}=\sigma_{3} / \tau$ is related to $Z(\phi)$ through

$$
P\left(I_{1}, I_{3}\right)=\int \frac{d \phi_{1}}{2 \pi} \frac{d \phi_{3}}{2 \pi} Z(\phi) e^{-i \tau\left(\phi_{1} I_{1}+\phi_{3} I_{3}\right)} .
$$

Since we are in the large $\tau$ limit, we can evaluate the integrals in the saddle-point approximation, and obtain

$$
P\left(I_{1}, I_{3}\right) \propto \exp \left[-E_{0}\left(\phi^{*}\right)-i \tau\left(\phi_{1}^{*} I_{1}+\phi_{3}^{*} I_{3}\right)\right],
$$

where $\phi_{a}^{*}$ satisfy the saddle point condition

$$
\begin{aligned}
& \left.\frac{\partial E_{0}}{\partial \phi_{1}}\right|_{\phi_{1}^{*}, \phi_{3}^{*}}+i I_{1}=0, \\
& \left.\frac{\partial E_{0}}{\partial \phi_{3}}\right|_{\phi_{1}^{*}, \phi_{3}^{*}}+i I_{3}=0 .
\end{aligned}
$$

Assuming that the solutions are much smaller than $1, \phi_{a}^{*}$ $\ll 1$, we have that the Hamiltonian can be rewritten, including terms up to second order in $\phi$, as

$$
\hat{H}(\phi)=\left[-\frac{1}{2 \tau_{c}} \frac{\partial^{2}}{\partial \Phi_{2}^{2}}+\frac{1}{2 \tau_{S}}\left(\phi_{1}^{2}+\phi_{3}^{2}+2 \phi_{1} \phi_{3} \cos \Phi_{2}\right)\right],
$$

where we introduced the average time between spin transfers, $\tau_{S}=\tau_{V} / \Sigma_{n} p_{n}$. We recognize the Hamiltonian for the Mathieu equation

$$
H_{M}=-\frac{\partial^{2}}{\partial v^{2}}+2 q \cos (2 v)
$$

Thus the ground-state energy depends on the lowest Mathieu characteristic function $a_{0}(q)$, with the coupling strength given by $q=4\left(\tau_{c} / \tau_{S}\right) \phi_{1} \phi_{3}$. Namely,

$$
E_{0}(\phi)=a_{0}(q) / 8 \tau_{c}+\left(\phi_{1}^{2}+\phi_{3}^{2}\right) / 2 \tau_{S} .
$$

The saddle-point equations (41) can then be combined to give a transcendent equation for $q$, from which one expresses $\phi_{a}^{*}$, which are purely imaginary, according to

$$
\begin{aligned}
& i \phi_{1}^{*}=\tau_{S} \frac{I_{1}-\left(I_{3} / 2\right) a_{0}^{\prime}\left(q^{*}\right)}{1-a_{0}^{\prime}\left(q^{*}\right)^{2} / 4}, \\
& i \phi_{3}^{*}=\tau_{S} \frac{I_{3}-\left(I_{1} / 2\right) a_{0}^{\prime}\left(q^{*}\right)}{1-a_{0}^{\prime}\left(q^{*}\right)^{2} / 4} .
\end{aligned}
$$

Here, $q^{*}$ is the solution to the equation

$$
\frac{q}{4}=-\frac{\left(\nu_{1}+\nu_{3}\right)^{2}}{\left[2+a_{0}^{\prime}(q)\right]^{2}}+\frac{\left(\nu_{1}-\nu_{3}\right)^{2}}{\left[2-a_{0}^{\prime}(q)\right]^{2}},
$$

where we introduced dimensionless currents $\nu_{a} \equiv \sqrt{\tau_{c} \tau_{S}} I_{a}$. Equations (43) and (44) are valid in the limit $\tau_{S} I_{a} \ll 1$, i.e., $\nu_{a} \ll \sqrt{\tau_{c} / \tau_{S}}$.

Finally, we have that the probability distribution is

$$
\begin{aligned}
\ln P\left(I_{1}, I_{3}\right) \propto & -a_{0}\left(q^{*}\right) / 8-\left(\nu_{1}+\nu_{3}\right)^{2}\left[1+a_{0}^{\prime}\left(q^{*}\right)\right] /\left[2+a_{0}^{\prime}\left(q^{*}\right)\right]^{2} \\
& -\left(\nu_{1}-\nu_{3}\right)^{2}\left[1-a_{0}^{\prime}\left(q^{*}\right)\right] /\left[2-a_{0}^{\prime}\left(q^{*}\right)\right]^{2} .
\end{aligned}
$$

This probability distribution is to be compared with the one predicted by applying the PP. The latter is, in the same regime $\tau_{S} I_{a} \ll 1$, the independent combination of two Gaussians:

$$
\ln P_{\mathrm{PP}}\left(I_{1}, I_{3}\right) \propto-\left(\nu_{1}^{2}+\nu_{3}^{2}\right) / 2,
$$

the proportionality constant $\left(\tau / \tau_{c}\right)$ being the same.

In the limit $\tau_{c} \ll \tau_{S}$, we have that Eqs. (45) and (46) coincide. However, by taking into account that the detectors have a finite decoherence time $\tau_{c}$, and that the time between spin 


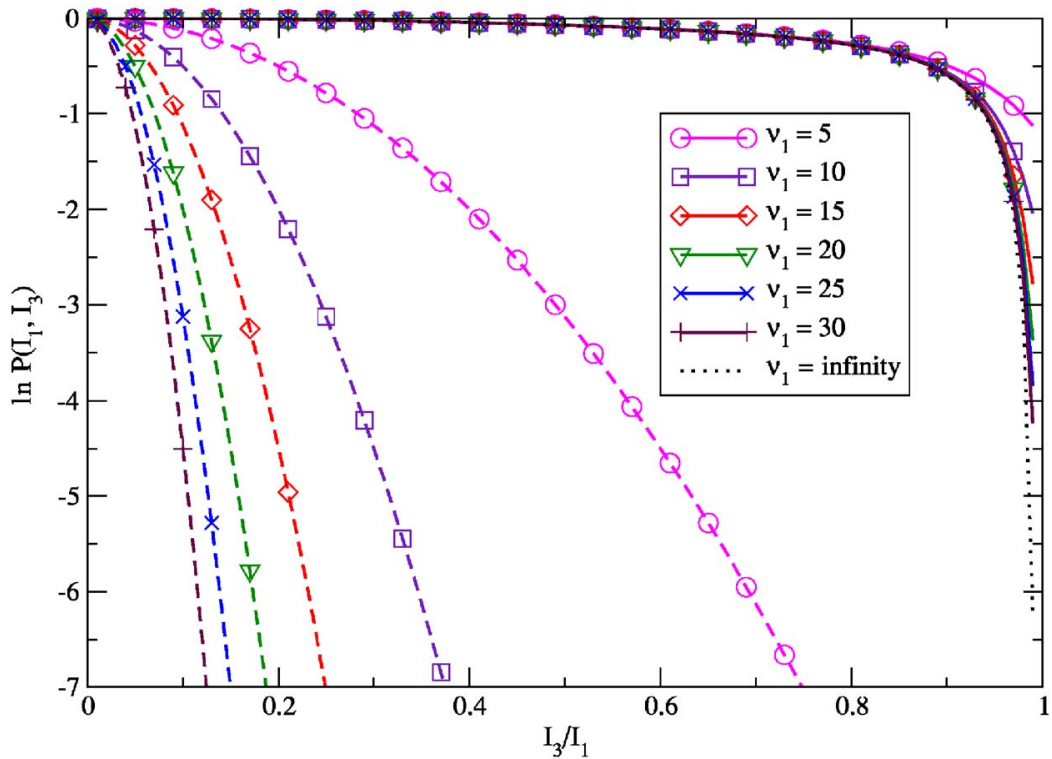

FIG. 3. (Color online) The log of probability as a function of $\nu_{3} / \nu_{1}$ for different values of $\nu_{1}$ for the configuration studied in the text. All the curves have been shifted by $\nu_{1}^{2} / 2$. The upper (full line) curves correspond to the result of the FCS approach, and the lower ones (dashed line) to the PP. The black dotted curve is the limiting scaling curve given by Eq. (47). transfers $\tau_{S}$ can be much smaller than $\tau_{c}$, we find that the probability distribution deviates sensibly from Eq. (46). This deviation is larger in the regime $1 \ll\left|\nu_{1}\right| \simeq\left|\nu_{3}\right| \ll \sqrt{\tau_{c} / \tau_{S}}$. when both (dimensionless) currents are comparable in module and large with respect to 1 . We stress that the correlation time is $\tau_{V}$, and, for a many channel case, it is possible to have $\tau_{S} \ll \tau_{V}$. Thus, the deviation from PP appears even if the experimenter chooses the response time of the detector $\tau_{c}$ $<\tau_{V}$, as it is sensible to do. When $\nu_{1} \gg 1$, we find that

$$
\ln P \propto-\nu_{1}^{2} / 2+f\left(\nu_{3} / \nu_{1}\right),
$$

with the scaling function $f(x)$ defined by

$$
f(x)=-\frac{a_{0}\left[q_{0}(x)\right]}{8}+\frac{1}{4} x q_{0}(x),
$$

where the condition $\partial a_{0} /\left.\partial q\right|_{q=q_{0}}=2 x$ defines $q_{0}(x)$. In particular, $f(x)$ diverges at $x=1$ according to $f(x) \simeq-1 / 16(1$ $-x)$. In Fig. 3 we draw the logarithm of probability as a function of $\nu_{3} / \nu_{1}$ for several values of $\nu_{1}$, and compare with the probability predicted by making use of PP.

\section{CONCLUSIONS}

We have discussed the full counting statistics of noncommuting variables. As a concrete example, we focused on spin counts in a two terminal device with nonferromagnetic leads connected through a nonpolarizing coherent conductor. We have provided a formula connecting the FCS of spins to the one of charge. We have seen that it is crucial to have a coherent conductor with finite transparency connecting the two leads. This is because electrons transmitted through the same channel are in a spin singlet, and thus contribute no net spin transfer nor spin fluctuations. However, if the transmission probability through channel $n$ is finite $\left(0<T_{n}<1\right)$, then there is a nonzero probability $p_{n}=2\left(1-T_{n}\right) T_{n}$ that exactly one electron out of a singlet pair is transmitted, and this contributes to spin fluctuations.

Another interesting conclusion which we can draw from this work is that, when measuring noncommuting quantities with subsequent detectors, one should take into account the quantum dynamics of the detectors themselves. This is because the decoherence time for the detectors $\tau_{c}$ can be larger than the average time between two subsequent counts $\tau_{S}$. Thus if one would naively apply the projection postulate to the system after each count, the wrong prediction would be obtained. We have shown that, in the system considered here, such a deviation from the naïve application of the projection postulate is revealed by the fourth correlator of spin counts.

\section{ACKNOWLEDGMENTS}

A.D.L. thanks D. V. Averin for discussions and for pointing out Refs. 20 and 21. A.D.L. and Yu.V.N. acknowledge the financial support provided through the European Community's Research Training Networks Programme under Contract No. HPRN-CT-2002-00302, Spintronics.

\footnotetext{
*Current address: Department of Physics and Astronomy, Stony Brook University, SUNY, Stony Brook, NY 11794-3800.

${ }^{1}$ Y. M. Blanter and M. Büttiker, Phys. Rep. 336, 1 (2000).

${ }^{2}$ L. S. Levitov, H. W. Lee, and G. B. Lesovik, J. Math. Phys. 37, 4845 (1996).
}

${ }^{3}$ L. S. Levitov and G. B. Lesovik, JETP Lett. 58, 230 (1993).

${ }^{4}$ L. V. Keldysh, Zh. Eksp. Teor. Fiz. 67, 1515 (1964).

${ }^{5}$ Y. V. Nazarov, Ann. Phys. SI-193, 507 (1999).

${ }^{6}$ W. Belzig and Y. V. Nazarov, Phys. Rev. Lett. 87, 197006 (2001).

${ }^{7}$ W. Belzig, in Quantum Noise in Mesoscopic Physics, edited by Y. 
V. Nazarov (Kluwer, Dordrecht, 2002).

${ }^{8}$ F. Pistolesi, Phys. Rev. B 69, 245409 (2004).

${ }^{9}$ A. Romito and Y. V. Nazarov, Phys. Rev. B 70, 212509 (2004).

${ }^{10}$ A. V. Andreev and E. G. Mishchenko, Phys. Rev. B 64, 233316 (2001).

${ }^{11}$ J. C. Cuevas and W. Belzig, Phys. Rev. Lett. 91, 187001 (2003).

${ }^{12}$ G. Johansson, P. Samuelsson, and A. Ingerman, Phys. Rev. Lett. 91, 187002 (2003).

${ }^{13}$ Y. V. Nazarov and D. A. Bagrets, Phys. Rev. Lett. 88, 196801 (2002).

${ }^{14}$ A. Di Lorenzo and Y. V. Nazarov, Phys. Rev. Lett. 93, 046601 (2004)

${ }^{15}$ Y. V. Nazarov and M. Kindermann, Eur. Phys. J. B 35, 413 (2003).

${ }^{16}$ J. von Neumann, Mathematical Foundations of Quantum Mechanics (Princeton University Press, Princeton, 1932).

${ }^{17}$ Semiconductor Spintronics and Quantum Computation, edited by D. D. Awschalom, D. Loss, and N. Samarth (Springer, Berlin, 2002).

${ }^{18}$ C. Chang, Proc. IEEE 91, 1756 (2003).
${ }^{19}$ G. B. Lesovik and R. Loosen, JETP Lett. 65, 295 (1997); G. B. Lesovik, Phys. Usp. 41, 145 (1998); D. V. Averin, in Exploring the Quantum/Classical Frontier: Recent Advances in Macroscopic Quantum Phenomena, edited by J. R. Friedman and S. Han (Nova Science Publishers, New York, 2002).

${ }^{20}$ A. N. Korotkov, in Quantum Noise in Mesoscopic Physics, edited by Y. V. Nazarov (Kluwer, Dordrecht, 2002).

${ }^{21}$ J. Dalibard, Y. Castin, and K. Mølmer, Phys. Rev. Lett. 68, 580 (1992).

${ }^{22}$ R. Feynman and F. Vernon, Ann. Phys. (N.Y.) 24, 218 (1963).

${ }^{23}$ H. Kleinert, Path Integrals in Quantum Mechanics, Statistics, Polymer Physics, and Financial Markets, 3rd ed. (World Scientific, Singapore, 2002).

${ }^{24}$ A. Cimmino, G. I. Opat, A. G. Klein, H. Kaiser, S. A. Werner, M. Arif, and R. Clothier, Phys. Rev. Lett. 63, 380 (1989).

${ }^{25}$ Y. Aharonov and A. Casher, Phys. Rev. Lett. 53, 319 (1984).

${ }^{26}$ O. Costa de Beauregard, Phys. Lett. 24A, 177 (1967).

${ }^{27}$ G. P. Fisher, Am. J. Phys. 39, 1528 (1971).

${ }^{28}$ G. B. Lesovik and N. M. Chtchelkatchev, JETP Lett. 77, 393 (2003). 